\title{
Analytical Study of Thermal Heat Conduction inside Two Orthotropic Mediums in Cylindrical Geometry
}

\author{
Mohammed Nikchi, Hamid Hamza, Khalid Zniber, Jawad Lahjomri and Abdelaziz Oubarra \\ Laboratory of Mechanics, Faculty of Science Aïn Chock, University Hassan II, \\ Casablanca 20100, Morocco \\ mohammed.nikchi-etu@etu.univh2c.ma
}

\begin{abstract}
The present work focuses on the two-dimensional steady-state thermal behavior of two orthotropic mediums in a coaxial cylindrical geometry. The whole lateral surface is subjected to a flux density while the end sections are maintained at prescribed temperatures. The resolution of the problem is carried out analytically by the variable separation technique, taking into account the boundary conditions and the continuity of temperatures and flux at the interface between the two mediums. The resolution in dimensionless form, expressed in terms of the Bessel functions, made it possible to identify the parameters of the study, namely the ratio of the main thermal conductivities for each medium as well as the ratio of the radial thermal conductivities of the two mediums.

A numerical code was developed, in order to simulate the effect of the study parameters on heat transfer within the two mediums, in terms of isotherms.
\end{abstract}

Keywords: Orthotropic medium, Variable separation method, Bessel function, Axial thermal conductivity, Radial thermal conductivity, Isotherms.

\section{Introduction}

Composite materials are widely used in heat transfer and thermal management of equipment, such as aerospace [1-3], braking and friction systems [4,5], fins, tanks, heat exchangers [6-9], electrical applications [10,11] and biomaterials [12,13]. The effective thermal conductivity is one of the main thermo-physical properties used to quantify the thermal behavior of these heterogeneous materials. This property is strongly affected by the composition and structure of these materials $[14,15]$.

Several studies have been made in the case of orthotropic medium, among others Haji-Sheikh et al who studied the steady [17] and unsteady [18] thermal behavior of a two-slab 3D body, by the resolution of the steady-state multidirectional heat equation using technique of separate variables. They showed that steady-state solution of a temperature field in a multilayer body is an invaluable tool for analyzing heat spreaders in electronic cooling applications. This includes heat spreaders with orthotropic or isotropic layers. The study of A. Amiri Delouei et al [19] presents an exact analytical solution of transient heat conduction in cylindrical multilayer composite laminates. This solution is valid for the most generalized linear boundary conditions consisting of the conduction, convection and radiation heat transfer. Here, it is supposed that the fibers are winded around the cylinder and their direction can be changed in each lamina. Laplace transformation is applied to change the domain of the solutions from time into the frequency. An appropriate Fourier transformation has been derived using the Sturm-Liouville theorem. The results obtained in this investigation can be employed for predicting and controlling the thermal stress and thermal fraction in composite structures. In the same context, M.H. Kayhani et al [20] present a steady analytical solution for heat conduction in a cylindrical multilayer composite laminate in which the fiber direction may vary between layers. The analytical solution is obtained for general linear boundary conditions that are suitable for various conditions including combinations of conduction, convection, and radiation both inside and outside the cylinder. The Sturme-Liouville theorem is used to derive an appropriate Fourier transformation for this problem. The analytical solution can be used for a wide range of thermal boundary conditions, which includes many applied problems.

The present paper, steady heat conduction in two orthotropic cylinders is investigated analytically. Like A. Amiri Delouei et al [19] and M.H. Kayhani et al [20], we focus on symmetrical steady heat conduction in two orthotropic cylinders in longitudinal and radial directions $(r, z)$. The main innovation of the present study is the derivation of the most general analytical solution based on general boundary conditions, which are based on Dirichlet and Newman boundary conditions. For this purpose, we use the variable separation technique, taking into account the boundary conditions and the continuity of 
the temperatures and flux at the interface between the two mediums. This resolution in dimensionless form, expressed in terms of the Bessel functions, made it possible to identify the parameters of the study, namely the ratio of the main thermal conductivities for each medium as well as the ratio of the radial thermal conductivities of the two mediums.

\section{Problem Formulation and Mathematical Modelling}

The study concerns two orthotropic medium in coaxial cylindrical geometry of length $L$ (Figure 1). One of the medium symbolized by $A$ occupies the interior cylinder of radius $a$, while the second medium $B$ is confined in the annular space limited by the rays $a$ and $b$. The left and right ends of the two cylinder ( $z=0$ and $z=L$ ) are maintained at the same constant temperatures $\left(T_{0}\right)$, while the lateral surface $(r=b)$ is subjected to an imposed flux density.

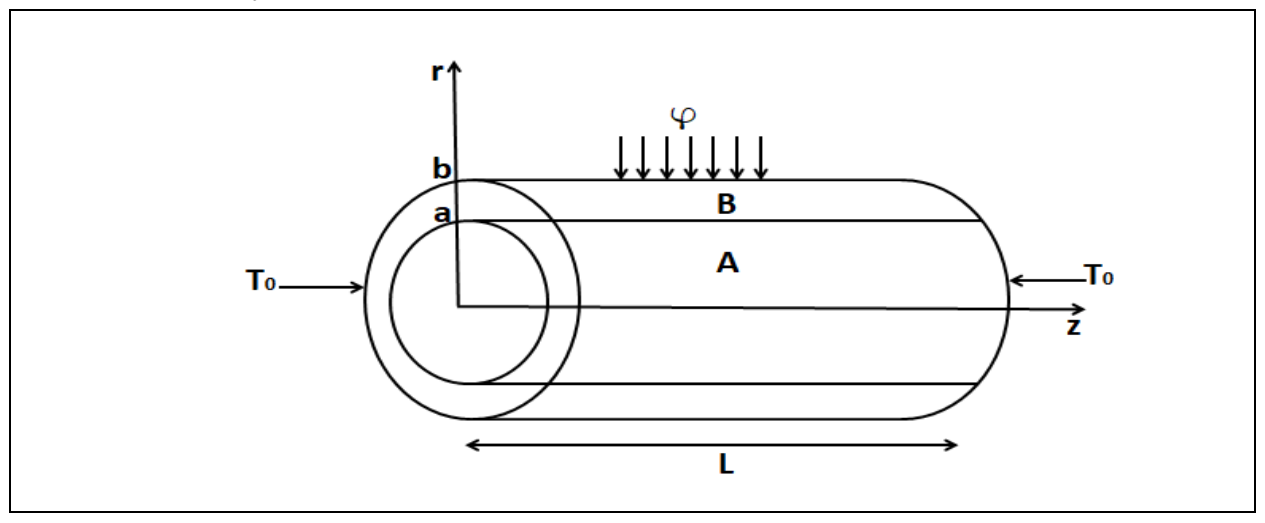

Fig. 1: Three-dimensional representation of the two orthotropic cylinders.

Based on the previous assumptions, the equations of the heat diffusion for the two orthotropic mediums are written as:

$$
K_{r r}^{i} \frac{\partial^{2} T^{i}}{\partial r^{2}}+\frac{K_{r r}^{i}}{r} \frac{\partial T^{i}}{\partial r}+K_{z z}^{i} \frac{\partial^{2} T^{i}}{\partial z^{2}}=0
$$

The above equation is applicable respectively for the two orthotropic medium ( $i=A$ and $B$ ). $K_{r r}^{i}$ and $K_{z z}^{i}$ are the main thermal conductivities along the $r, z$ axes.

The governing differential equations for the temperature distribution are associated to the boundary and interfaces equations :

$$
\begin{array}{lc}
0 \leq r \leq b & T(z=0, r)=T_{0} \\
0 \leq r \leq b & T(z=L, r)=T_{0} \\
(r=0): & \left.\frac{\partial T^{A}}{\partial r}\right|_{r=0}=0 \\
(r=b): & \left.\frac{\partial T^{B}}{\partial r}\right|_{r=b}=\frac{\varphi}{k_{r r}^{B}} \\
(r=a): & \left.T^{A}(z, r)\right|_{r=a}=\left.T^{B}(z, r)\right|_{r=a}
\end{array}
$$




$$
(r=a):\left.\quad \frac{\partial T^{A}}{\partial r}\right|_{r=a}=\left.\frac{1}{\delta} \cdot \frac{\partial T^{B}}{\partial r}\right|_{r=a}
$$

Where, $\delta=\frac{k_{r r}^{A}}{k_{r r}^{B}}$.

The above equations (1)-(7) can be cast in dimensionless form (8)-(14) by incorporating the following dimensionless parameters:

$$
Z=\frac{z}{b} \quad R=\frac{r}{b} \quad \Theta^{i}=\frac{T^{i}-T_{0}}{T_{0}} \quad \Phi_{R, Z}^{i}=\frac{\varphi_{r, z}^{i}}{\varphi_{\operatorname{Re} f}}
$$

Where, $\varphi_{\operatorname{Re} f}=K_{z z}^{B} \frac{T_{0}}{L}$.

$$
\frac{\partial^{2} \Theta^{i}}{\partial R^{2}}+\frac{1}{R} \frac{\partial \Theta^{i}}{\partial R}+\frac{K_{z z}^{i}}{K_{r r}^{i}} \cdot \frac{\partial^{2} \Theta^{i}}{\partial Z^{2}}=0
$$

With; $\frac{k_{z z}^{i}}{k_{r r}^{i}}$ Main thermal conductivity ratio, remains the same for the two mediums in the study of this problem. It will be expressed simply by $\frac{k_{z z}}{k_{r r}}$.

$$
\begin{array}{lcc}
(Z=0): & \Theta^{i}(Z=0, R)=\Theta_{l}^{i}=0 & 0 \leq R \leq 1 \\
(Z=G): & \Theta^{i}(Z=G, R)=\Theta_{r}^{i}=0 & 0 \leq R \leq 1 \\
(R=0): & \left.\frac{\partial \Theta^{A}}{\partial R}\right|_{R=0}=0 & 0 \leq Z \leq G \\
(R=1): & \left.\frac{\partial \Theta^{B}}{\partial R}\right|_{R=1}=\frac{v^{2}}{G} \Phi & 0 \leq Z \leq G \\
(R=\xi): & \left.\Theta^{A}(Z, R)\right|_{R=\xi}=\left.\Theta^{B}(Z, R)\right|_{R=\xi} & 0 \leq Z \leq G \\
(R=\xi): & \left.\frac{\partial \Theta^{A}}{\partial R}\right|_{R=\xi}=\left.\frac{1}{\delta} \cdot \frac{\partial \Theta^{B}}{\partial R}\right|_{R=\xi} & 0 \leq Z \leq G
\end{array}
$$

Where, $\xi=\frac{a}{b}$.

Solving the above equation system provides access to the temperature profile in the two mediums involved according to the $Z$ and $R$ directions cylindrical coordinates. 


\section{Analytical Solution}

The resolution of the problem is carried out analytically by the variable separation technique, taking into account the boundary conditions and the continuity of the temperatures and flux at the interface between the two mediums.

The first step is the use of the boundary conditions (equations 9-12), leading to an intermediate solution of the dimensionless temperatures of medium $A$ and $B$ in the following forms:

$$
\begin{gathered}
\Theta^{A}(R, Z)=\sum_{n=1}^{\infty} A_{n} \sin \left(\frac{n \pi}{G} Z\right) \cdot I_{0}\left(\frac{n \pi}{G} R\right) \\
\Theta^{B}(R, Z)=\sum_{n=1}^{\infty}\left[\frac{v}{n \pi} \cdot \frac{I_{0}\left(\frac{n \pi v}{G} R\right)}{I_{1}\left(\frac{n \pi v}{G}\right)}+D_{n}\left(\frac{K_{1}\left(\frac{n \pi v}{G}\right)}{I_{1}\left(\frac{n \pi v}{G}\right)} \cdot I_{0}\left(\frac{n \pi v}{G} R\right)+K_{0}\left(\frac{n \pi v}{G} R\right)\right] \cdot \sin \left(\frac{n \pi}{G} Z\right)\right.
\end{gathered}
$$

Where, $v=\sqrt{\frac{k_{z z}}{k_{r r}}}$.

The problem constants $A_{n}$ and $D_{n}$ are determined by the condition of continuity of the temperature and the flux at the interface $\xi=\frac{a}{b}$. (equations 13 and 14). This makes it possible to establish the final expressions of dimensionless temperatures of medium $A$ and $B$ in the following forms:

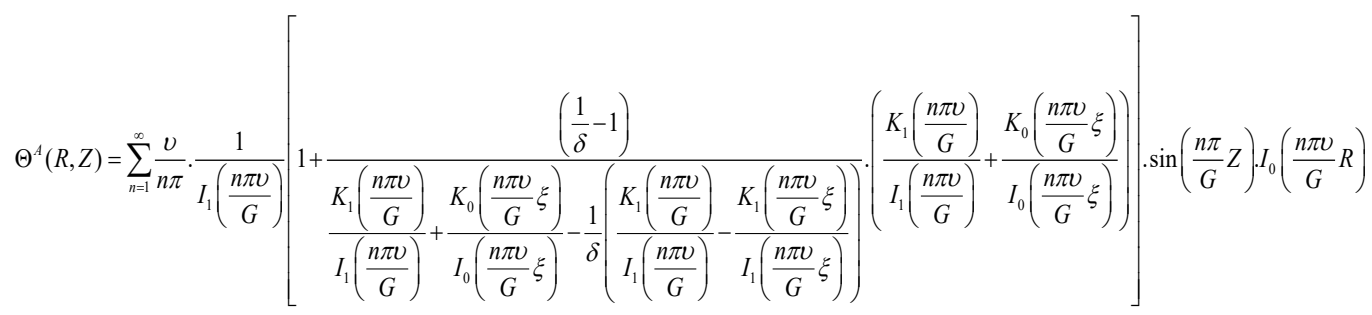

$$
\begin{aligned}
& \Theta^{B}(R, Z)=\sum_{n=1}^{\infty} \frac{v}{n \pi} \cdot \frac{1}{I_{1}\left(\frac{n \pi v}{G}\right)}\left[I_{0}\left(\frac{n \pi v}{G} R\right)+\frac{\left(\frac{1}{\delta}-1\right)}{\frac{K_{1}\left(\frac{n \pi v}{G}\right)}{I_{1}\left(\frac{n \pi v}{G}\right)}+\frac{K_{0}\left(\frac{n \pi v}{G} \xi\right)}{I_{0}\left(\frac{n \pi v}{G} \xi\right)}-\frac{1}{\delta} \cdot\left(\frac{K_{1}\left(\frac{n \pi v}{G}\right)}{I_{1}\left(\frac{n \pi v}{G}\right)}-\frac{K_{1}\left(\frac{n \pi v}{G} \xi\right)}{I_{1}\left(\frac{n \pi v}{G} \xi\right)}\right)} \cdot\left(\frac{K_{1}\left(\frac{n \pi v}{G}\right)}{I_{1}\left(\frac{n \pi v}{G}\right)} I_{0}\left(\frac{n \pi v}{G} R\right)+K_{0}\left(\frac{n \pi v}{G} R\right)\right)\right] \cdot \sin \left(\frac{n \pi}{G} Z\right)
\end{aligned}
$$

The temperatures within each medium defined by equations 17 and 18 are determined according to an iterative calculation, by comparing the successive all inside temperatures difference between two serial order $n$ and $n+1$ with a criterion of convergence $\varepsilon$, according to :

$$
\left|\Theta^{\mathrm{A}}(R, Z, n+1)-\Theta^{\mathrm{A}}(R, Z, n)\right|+\left|\Theta^{\mathrm{B}}(R, Z, n+1)-\Theta^{\mathrm{B}}(R, Z, n)\right|<\varepsilon
$$




\section{Problem Data}

The study is conducted for two orthotropic mediums in cylindrical geometry supposed of a form factor $G=2$, submitted submitted to a sinusoidal flux $\left(\Phi=\sin \left(\frac{\pi Z}{G}\right)\right)$ on the lateral surface. The mediums are characterized by main thermal conductivities according the axial and radial direction. The effect of these parameters is represented by the values of radial radial thermal conductivities ratio of the two mediums, $\delta=\frac{k_{r r}^{A}}{k_{r r}^{B}}$, taking values equal to $0.5,1,2$ and by the ratio between axial and radial conductivity $\frac{k_{z z}}{k_{r r}}$ equal to $0.25,1,4$. The orthotropic mediums have a symmetrical configuration in term of axial boundary conditions $\Theta(Z=0, R)=0, \Theta(Z=1, R)=0$. Interface location between the mediums is taking equal to $\xi=0.8$.

\section{Results and Discussion}

Figure 2 show the steady state temperature distribution within the two mediums for the various values of the study parameters. For each figure, we have simultaneously the domains associated to the $A$ and $B$ mediums separated by the interface characterized by $\xi=\frac{a}{b}$. A horizontal reading allows to deduce the impact of main thermal conductivities $\frac{k_{z z}}{k_{r r}}$ for each medium, while vertically, we get radial conductivities ratio effect $\delta$. For all the figures we note isothermal symmetry according to boundary conditions. Isotherms thermal levels are higher in the middle of lateral surface, subjected to a sinusoidal density flux. Two intermediates cases are present $\frac{k_{z z}}{k_{r r}}=1$ (the mediums became isotropic), and $\delta=1$ (The mediums have the same radial conductivities). For simultaneously $\frac{k_{z z}}{k_{r r}}=1$ and $\delta=1$, the two mediums are reduced to a single one. Apart from these intermediates configurations, isothermal profile has two aspects. For low value of $\frac{k_{z z}}{k_{r r}}=0.25$ corresponding to weak mediums axial conductivities, isotherms are parallel to cylinder sections except in the center, where the shape of the isotherms translates the heat sinusoidal flux applied to the outer surface. The increase of radial conductivities ratio $\delta$, that is to say increasing radial conductivity of medium $A$, reduce thermal level of temperature inside the two medium and make the isothermal profiles less tight. For high medium axial conductivities $\frac{k_{z z}}{k_{r r}}=4$, thermal level of the isotherms is higher than before. The isotherms are practically circular inside medium $B$ and penetrate advantage inside the second medium $A$, while being more and more spaced. Effect of radial conductivities ratio $\delta$ is not changed. 


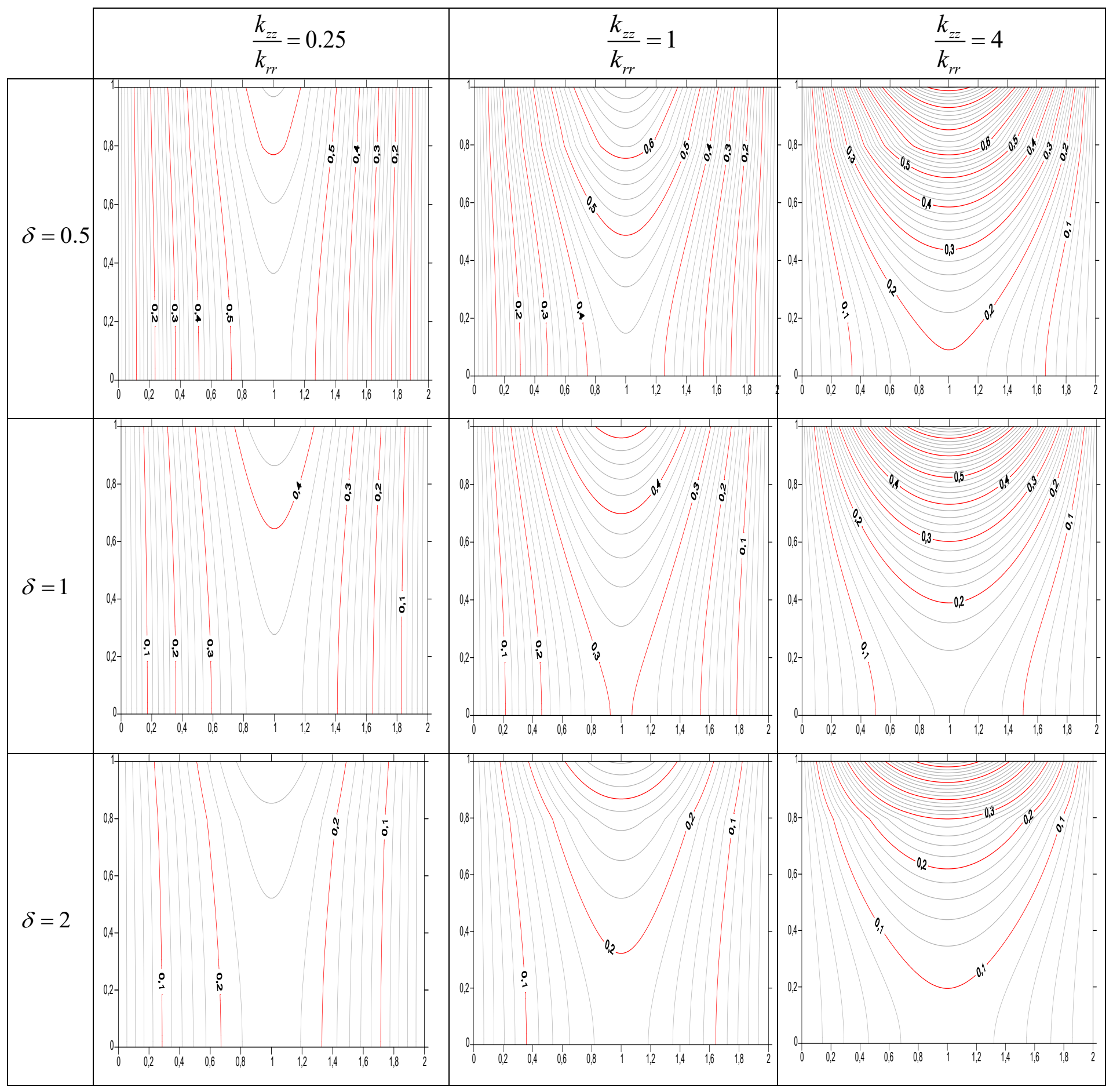

Fig. 2: Effect of study parameters on isotherms 


\section{Conclusion}

The work carried out relates to the analytical determination of the temperature field in two-dimensional steady-state orthotropic mediums. The cylindrical geometry is such that the lateral surface is subjected to a flux density while the sections sections are isothermal.

The main conclusions of this study are:

$\checkmark$ The isotherms are regular with no change of curvature at the interface for the ratio of radial thermal conductivities

$$
\delta=\frac{k_{r r}^{A}}{k_{r r}^{B}} \text { equal to } 1
$$

The isotherms are practically circular inside medium $B$ and penetrate advantage inside the second medium $A$, while being more and more spaced.

$\checkmark$ When the main thermal conductivity ratio $\frac{k_{z z}}{k_{r r}}$ increases for a constant radial thermal conductivity ratio $\delta=\frac{k_{r r}^{A}}{k_{r r}^{B}}$, the thermal level decreases.

$\checkmark$ The same thing happens when the radial thermal conductivity ratio $\delta=\frac{k_{r r}^{A}}{k_{r r}^{B}}$ increases for a constant main thermal conductivity ratio $\frac{k_{z z}}{k_{r r}}$, the thermal level decreases.

\section{NOMENCLATURE}

$\begin{array}{lll}a & : & \text { Radius of the inner cylinder [m] } \\ b & : & \text { Radius of the outer cylinder [m] } \\ A & : & \text { Inner orthotropic medium } \\ B & : & \text { Outer orthotropic medium } \\ G & : & \text { Form factor } \\ I_{0} & : & \text { Bessel functions of the first kind of order } 0 \\ I_{1} & : & \text { Bessel functions of the first kind of order } 1 \\ K_{0} & : & \text { Bessel functions of the second kind of order } 0 \\ K_{1} & : & \text { Bessel functions of the second kind of order } 1 \\ k_{r r} & : & \text { Thermal conductivity in the } \mathrm{r} \text { direction }[\mathrm{W} / \mathrm{m} . \mathrm{K}] \\ k_{z z} & : & \text { Thermal conductivity in the } \mathrm{z} \text { direction }[\mathrm{W} / \mathrm{m} . \mathrm{K}] \\ L & : & \text { Cylinder length [m] } \\ r & : & \text { Dimensional radial axis [m] } \\ R & : & \text { Dimensionless radial axis } \\ T & : & \text { Dimensional temperature [K] } \\ T_{0} & : & \text { Temperature at cylinder sections [K] }\end{array}$




$\begin{array}{lll}z & : & \text { Dimensional axial axis }[\mathrm{m}] \\ Z & : & \text { Dimensionless axial axis } \\ \delta & : & \text { Radial thermal conductivity ratio } \\ \Theta & : & \text { Dimensionless temperature }\end{array}$

\section{References}

[1] W. Krenkel, F. Berndt, C/C-SiC composites for space applications and advanced friction systems, Mater. Sci. Eng., A 412 (2005) 177-181.

[2] J. Jedidi, F. Jacquemin, A. Vautrin, Design of accelerated hygrothermal cycles on polymer matrix composites in the case of a supersonic aircraft, Compos. Struct. 68 (2005) 429-437.

[3] C.J. von Klemperer, D. Maharaj, Composite electromagnetic interference shielding materials for aerospace applications, Compos. Struct. 91 (2009) 467-472.

[4] P. Filip, Z. Weiss, D. Rafaja, On friction layer formation in polymer matrix composite materials for brake applications, Wear 252 (2002) 189-198.

[5] Z. Stadler, K. Krnel, T. Kosmac, Friction and wear of sintered metallic brake linings on a C/C-SiC composite brake disc, Wear 265 (2008) 278-285.

[6] Y. Xia, A.M. Jacobi, An exact solution to steady heat conduction in a twodimensional

slab on a one-dimensional fin: Application to frosted heat exchangers, Int. J. Heat Mass Transfer 47 (2004) 3317-3326.

[7] X. Fang, Z. Zhang, Z. Chen, Study on preparation of montmorillonite-based composite phase change materials and their applications in thermal storage building materials, Energy Convers. Manage. 49 (2008) 718-723.

[8] S. Pashah, A.F.M. Arif, Syed M. Zubair, Study of orthotropic pin fin performance

through axisymmetric thermal non-dimensional finite element, Appl. Therm. Eng. 31 (2011) 376-384.

[9] M.T. Mustafa, Syed.M. Zubair, A.F.M. Arif, Thermal analysis of orthotropic annular fins with contact resistance: A closed-form analytical solution, Appl. Therm. Eng. 31 (2011) 937-945.

[10] L.A. Dobrzanski, M. Drak, B. Ziebowicz, New possibilities of composite materials application-Materials of specific magnetic properties, J. Mater. Process. Technol. 191 (2007) 352-355.

[11] R. Olivé-Monllau, M.J. Esplandiu, J. Bartrolí, M. Baeza, F. Céspedes, Strategies for the optimization of carbon nanotube/polymer ratio in composite materials: Applications as voltammetric sensors, Sensors Actuat. B 146 (2010) 353-360.

[12] K. Fujihara, K. Teo, R. Gopal, P.L. Loh, V.K. Ganesh, S. Ramakrishna, K.W.C. Foong, C.L. Chew, Fibrous composite materials in dentistry and orthopaedics: Review and applications, Compos. Sci. Technol. 64 (2004) 775-788.

[13] E. Stodolak, T. Gumula, R. Leszczynski, J. Wieczorek, S. Blazewicz, A composite material used as a membrane for ophthalmology applications, Compos. Sci. Technol. 70 (2010) 1915-1919.

[14] C. PROFETA and M. THOUVENIN, "Mesure Des Propriétés Thermophysiques," Projet de mécanique 1ère année Ecole des Mines de Nancy, pp. 1-25, 2004.

[15] D. M. Bigg, "Thermal Conductivity of heterophase Polymer Compositions," Advances in Polymer Science, vol. 119, pp. 1-30, 1995.

[16] Y. Zhang, A. Fan, S. Luo, H. Wang, Suspended 2D anisotropic materials thermal diffusivity measurements using dualwavelength flash Raman mapping method, Int. J. Heat Mass Transfer, 145 (2019) 118795.

[17] A.Haji-Sheikh, J.V. Beck, D. Agonafer, Steady-state heat conduction in multi-layer bodies, Int. J. Heat Mass Transfer 46(2003) 2363-2379.

[18] A. Haji-Sheikh, J.V. Beck, D. Agonafer, Temperature solution in multidimensional multi-layer bodies, Int. J. Heat Mass Transfer 45 (2002) 1865-1877.

[19] H. Kayhani, M. Norouzi, A. Amiri Delouei, Int. J. of Thermal Sciences 52 (2012) 73-82.

[20] A. Amiri Delouei, M. H. Kayhani, M. Norouzi, Int. J. of Heat and Mass Transfer 55 (2012) 4427-4436. 